\title{
Lowered LDL-C Levels Reduce Later Local Vascular Events after Surgical or Endovascular Treatment of Peripheral Artery Disease
}

\author{
Kouji Ishii, MD, ${ }^{1}$ Junichiro Takahashi, MD, ${ }^{1}$ Tsuyoshi Kanaoka, MD, ${ }^{1}$ Yutaka Wakamatsu, MD, ${ }^{1}$ \\ Toshihiro Gohda, MD, ${ }^{1}$ Shigeyuki Sasaki, MD, ${ }^{2}$ and Yoshiro Matsui, $\mathrm{MD}^{3}$
}

Purpose: To examine the relationship between incidence of later, local vascular events (restenosis and occlusion) and clinical factors including lipid levels after surgical or endovascular treatment of peripheral artery disease (PAD).

Methods: Consecutive 418 PAD lesions (in 308 patients under the age of 70) treated with surgical $(n=188)$ or endovascular $(n=230)$ repair for iliac $(n=228)$ and infrainguinal $(n=190)$ lesions were retrospectively analyzed. Clinical features and lipid levels were compared between patients who developed vascular events ( $=51$; VE group) and those who did not ( $n=257$; NoVE group).

Results: Among assessed factors, post-therapeutic low-density lipoprotein cholesterol (LDL-C) levels $(\mathrm{mg} / \mathrm{dL})$ were significantly higher in the VE group $(120.4 \pm 31.2)$ than in the NoVE group $(108.2 \pm 25.1)$ $(P=0.01)$. Infrainguinal lesions were more common in the $V E$ than in the NoVE group $(P<0.001)$. Cox hazard analysis indicated that infrainguinal lesions relative to iliac lesions significantly increased the risk of vascular events (hazard ratio (HR) $3.35 ; 95 \%$ CI 1.63-6.90; $P=0.001$ ) and post-therapeutic LDL-C levels $<130(\mathrm{mg} / \mathrm{dL}$ ) decreased the risk (HR $0.34 ; 95 \%$ I $0.17-0.67 ; P=0.002)$.

Conclusion: Lowered post-therapeutic LDL-C levels can decrease the risk of later, local vascular events after PAD treatment. These results may support the rationale for aggressive lipid-modifying therapy for PAD.

Keywords: dyslipidemia, LDL-C, PAD, endovascular treatment, surgical treatment

\section{INTRODUCTION}

$\mathrm{P}$ eripheral artery disease (PAD) due to atherosclerosis occurs commonly, with an estimated prevalence as high as $21 \%$ in patients aged over 65 years in the Western

\footnotetext{
${ }^{1}$ Division of Cardiovascular Surgery, Aishin Memorial Hospital, Sapporo, Hokkaido, Japan

${ }^{2}$ Division of Medical Science, Health Sciences University of Hokkaido, Ishikari-tobetsu, Hokkaido, Japan

${ }^{3}$ Department of Cardiovascular Surgery, Hokkaido University

Hospital, Sapporo, Hokkaido, Japan
}

Received: January 5, 2012; Accepted: May 6, 2012

Corresponding author: Shigeyuki Sasaki, MD. Division of Medical Science, Health Sciences University of Hokkaido, Kanazawa 1757, Ishikari-Tobetsu, Hokkaido 061-0212, Japan

Tel: +81-133-23-1852, Fax: +81-133-23-1852

E-mail: sasakish@hoku-iryo-u.ac.jp world. ${ }^{1-4)}$ Symptoms of PAD range from claudication to rest pain or tissue loss, and patients presenting with chronic critical limb ischemia (CLI) are frequently associated with other arteriosclerotic cardiovascular disorders like coronary artery disease (CAD). ${ }^{2,3,5)}$ Thus, reductions in atherosclerotic risk factors, such as smoking cessation, treatment for diabetes and dyslipidemia, play an important role in the treatment of PAD as well as treatment for affected arteries. ${ }^{6,7)}$ The established American Heart Association guidelines recommend the use of hydroxymethylglutaryl (HMG) coenzyme-A reductase inhibitors (statin drugs), angiotensin converting enzyme (ACE) inhibitors, and antiplatelet agents in patients with systemic atherosclerosis, such as those undergoing operative intervention to treat PAD. ${ }^{6-8)}$

Currently, most patients with PAD are treated on the 
basis of the Trans-Atlantic Inter-Society Consensus document on Management of Peripheral Arterial Disease (TASC) and the Inter-Society Consensus for the Management of Peripheral Arterial Disease (TASC II) Steering Committee guidelines. ${ }^{9,10)}$ Long-term results after treatment of PAD, however, may vary with the type and extent of the lesions and the presence of atherosclerotic risk factors and comorbidity. The reported predictors for adverse outcomes after treatment of PAD include the TASC type C/type D lesions, stenosis of the ipsilateral peripheral artery, smoking history, diabetes mellitus (DM), chronic renal failure with hemodialysis, and critical limb ischemia. ${ }^{6,11,12)}$ With regard to serum lipid levels, few reports have described the direct relationship between the lipid levels and long-term results of the affected arteries after surgical or endovascular treatment of PAD. ${ }^{6,13)}$ Although the relative benefit of lipid-modifying therapies for claudication symptoms remains unclear, ${ }^{6)}$ the hypothesis that lowered low-density lipoprotein cholesterol (LDL-C) levels are beneficial in promoting graft patency in surgically treated cases and decreasing local vascular events (restenosis and occlusion) in patients with PAD is logical. It seems to be important to clarify the direct relationship between the lipid levels such as LDL-C and high-density lipoprotein cholesterol (HDL-C) and long-term local vascular events after surgical or endovascular treatment of PAD, as a background rationale for aggressive lipid-modifying therapy. For that purpose, we retrospectively analyzed the pre- and posttherapeutic serum lipid levels and other atherosclerotic risk factors in patients undergoing surgical bypass procedures or endovascular treatment (EVT) for PAD. The purpose of this study was to investigate the relationship between the incidence of later local vascular events (restenosis and occlusion) and clinical factors including lipid levels after surgical or endovascular treatment for PAD.

\section{Patients and Methods}

This retrospective study was performed on consecutive 418 PAD lesions of the lower extremity treated with surgical procedures $(\mathrm{n}=188)$ or EVT $(\mathrm{n}=230)$ in 308 patients under the age of 70, between April 1989 and November 2010 in the Division of Cardiovascular Surgery, Aishin Memorial Hospital. All patients were Japanese and consisted of 270 male and 38 female patients with a mean age of $62.3 \pm 6.0$ years (range from 50 to 69 years). Clinical symptoms of these patients included intermittent claudication (IC) for 260 (84.4\%) and CLI for 48 patients (15.6\%). Patients with IC were considered for surgical procedures or EVT in principle when medical therapies failed to significantly improve ischemic symptoms. The diagnosis of PAD was established by the findings of digital subtraction angiography (DSA) or computed tomographic angiography (CTA) in all patients, and arterial segments with greater than $75 \%$ diameter stenosis were determined as significant stenosis. Either before or after revascularization, 217 patients (70.5\%) received treatment for hypertension (HTN); 124 (40.3\%) for DM and 119 (38.6\%) received statin drugs for dyslipidemia. Of the 119 patients who received statin, 49 patients received statin drugs since before the treatment of PAD, and all 119 patients received after the treatment. Nineteen patients $(6.2 \%)$ needed hemodialysis due to chronic renal failure. Antiplatelet therapy or anticoagulant agents were given in principle to all patients, unless the patient presented with serious side effects. Due to the high annual mortality rate reported in patients with PAD, ranging from $4 \%$ to $6 \%$ and even as high as $25 \%$ in patients presenting with CLI, ${ }^{6}$ study subjects were limited to those under the age of 70 at treatment to ensure sufficient period of follow-up and to minimize the influence of aging on the local vascular events.

Of 418 lesions investigated in this study, there were 228 iliac lesions and 190 infrainguinal lesions. The iliac lesions included TASC II type A for 123, type B for 45 , type $\mathrm{C}$ for 13 , and type $\mathrm{D}$ for 47 lesions, and the infrainguinal lesions included TASC II type A for 53, type B for 10, type $\mathrm{C}$ for 97 , and type $\mathrm{D}$ for 30 lesions. ${ }^{9,10)}$ Of these, 188 lesions were treated with surgery, including TASC II type B for 11, type C for 109, and type D for 68 lesions, and 230 lesions were treated with EVT, including TASC II type A for 176, type B for 44, type C for 1, and type $\mathrm{D}$ for 9 lesions. Therapeutic strategy during the study period has been changed in response to progress in endovascular technology and publication of TASC and TASC II guidelines. $\left.{ }^{9},{ }^{10}\right)$ Before the Palmaz stent was available in the EVT, surgical procedures tended to be selected more often, mainly due to a relatively high incidence of restenosis occurrence after plain old balloon angioplasty (POBA). Since the publication of TASC II guidelines, most of TASC II type A and type B lesions in the iliac arteries have been treated with EVT and the number of patients undergoing infrainguinal surgical bypass has been diminished.

Among 188 lesions treated with open surgical procedures, prosthetic graft (Dacron or expanded polytetrafluoroethylene (ePTFE) graft) was used in 176 lesion and 
autologous saphenous vein graft in 12 lesions. In these lesions, the etiologic source of PAD was considered to be atherosclerosis in all cases. Around the same time as study period, there were 5 patients who developed an early graft failure within a month after surgery and one patient who died within a month. These patients were not included in this study, as the main purpose of this study was to evaluate long-term effects of lipid levels on local vascular events. Among 230 lesions treated with EVT, 149 lesions were treated concomitantly with stent placement (Palmaz stent for 68 lesions and nitinol shapememory alloy-recoverable technology (SMART) stent for 81 lesions) and other 81 lesions were treated with POBA without stent placement.

Follow-up of the patient after discharge has been conducted periodically in principle at the outpatient clinic in our institute. Blood pressure at the outpatient clinic over the course of follow-up was controlled with a target systolic pressure of less than $140 \mathrm{mmHg}$ and diastolic pressure of less than $90 \mathrm{mmHg}$. Patients with HTN received ACE inhibitors or angiotensin-II receptor blockers concurrently with additional oral calcium-channel blockers as appropriate. For the measurement of serum lipid levels, blood samples for biochemical assay were collected after an overnight fast, and the serum was analyzed for total cholesterol (TC), HDL-C, and triglycerides (TG) using automated assay. The LDL-C levels were calculated by Friedewald formula. The LDL-C levels during the followup period were in principle controlled with a target value of at least less than $130 \mathrm{mg} / \mathrm{dL}$ using statin drugs and/or fibrates on physician preference. Post-therapeutic lipid levels in patients were determined by the mean value of laboratory data obtained at least 3 months after surgical treatment or EVT. Patients who were referred to other hospitals or lost to follow-up within 3 months after discharge were not included in this study. During the study period, there were 29 patients under such conditions, most of whom were referred to their family doctor at a long distance on a good postoperative course. Two patients, however, were transferred due to cerebral infarction.

Local vascular events, defined as restenosis greater than $75 \%$ in diameter and occlusion in this study, was suspected by the recurrence of ischemic symptoms of the lower extremity or a significant decrease of $20 \%$ or more in ankle brachial index (ABI) compared to the previous levels and confirmed by DSA or CTA. Duplex ultrasound, which is a useful tool to provide graft surveillance after infrainguinal bypass with saphenous venous (but not prosthetic) conduit, ${ }^{6}$ had not been used as routine surveil- lance in this study, as the prosthetic graft was used in the majority of patients undergoing surgical procedures. Clinical features including the age and gender, atherosclerotic risk factors, and pre-therapeutic and post-therapeutic lipid levels were recorded and compared between the patients who developed later local vascular events ( $\mathrm{n}=51$; VE group) and those who did not develop events ( $n=257$; NoVE group). The clinical factors that may be related to the development of later vascular events were subjected to multivariate analysis to identify correlations with the later events occurrence. Kaplan-Meier method was used to evaluate the rate of freedom from local vascular events. The endpoint of this study was defined as the time at local vascular events or tissue loss occurrence, the time of death from any cause (regarded as censored case), or time at the end of follow-up for a maximum of 10 years. In patients with multiple local vascular events, the time at the first event was defined as the endpoint of the study. This study was approved by the Hospital Ethics Committee and all patients received a full explanation of surgical or endovascular treatment and related risks, and treatment strategy for the vascular lesions by physicians. The mean follow-up period until the endpoint of the study was $51.8 \pm 38.3$ (months) after surgical treatment of EVT for PAD.

\section{Statistical Analysis}

Descriptive statistics were used, and continuous variables are presented as the mean \pm standard deviation (SD) and compared using the Student's t-test for parametric and Mann-Whitney U-test for non-parametric analyses. Chi-square test was used for comparisons of categorical variables. The rate of freedom from local vascular events (VE-free rate) was calculated by the Kaplan-Meier method and comparisons between the two groups were performed by the logrank test. Cox proportional hazard model was applied to determine the effects of variables over time on local vascular events during the follow-up period. Differences were statistically considered significant at $P<0.05$. All statistical analysis was performed using Dr. SPSS II version 11.0.1 J (SPSS Inc., Chicago, Illinois).

\section{Results}

Overview of the clinical characteristics and atherosclerotic risk factors compared between the VE and NoVE group is summarized in Table 1. Later local vascular 
Table 1 Comparative studies between the patients who did and did not develop later vascular events

\begin{tabular}{|c|c|c|c|c|}
\hline & $\begin{array}{c}\text { Total } \\
(\mathrm{n}=308)\end{array}$ & $\begin{array}{l}\text { Vascular events }(+) \\
(\text { VE group; } \mathrm{n}=51)\end{array}$ & $\begin{array}{c}\text { Vascular events }(-) \\
\text { (NoVE group; } n=257 \text { ) }\end{array}$ & $P$ value \\
\hline Age (year) & $62.3 \pm 6.0$ & $62.8 \pm 5.4$ & $62.2 \pm 6.1$ & 0.533 \\
\hline Male/Female & $270 / 38$ & $42 / 9$ & $228 / 29$ & 0.207 \\
\hline HTN treatment (\%) & $217(70.5 \%)$ & $35(68.6 \%)$ & $182(70.8 \%)$ & 0.740 \\
\hline DM treatment (\%) & $124(40.3 \%)$ & $25(49.0 \%)$ & $99(38.5 \%)$ & 0.211 \\
\hline Statin drugs (\%) & $119(38.6 \%)$ & $21(26.3 \%)$ & $98(13.4 \%)$ & 0.753 \\
\hline PCI or CABG (\%) & $30(9.7 \%)$ & $2(3.9 \%)$ & $28(10.9 \%)$ & 0.193 \\
\hline Hemodyalysis (\%) & $19(6.2 \%)$ & $4(7.8 \%)$ & $15(5.8 \%)$ & 0.532 \\
\hline \multicolumn{5}{|l|}{ Pre-therapeutic lipid levels } \\
\hline HDL-C (mg/dL) & $43.6 \pm 13.0$ & $43.5 \pm 10.5$ & $43.6 \pm 13.5$ & 0.940 \\
\hline LDL-C (mg/dL) & $116.1 \pm 30.7$ & $119.2 \pm 36.4$ & $115.5 \pm 29.5$ & 0.439 \\
\hline LDL-C/HDL-C ratio & $2.90 \pm 1.17$ & $2.89 \pm 1.05$ & $2.91 \pm 1.19$ & 0.898 \\
\hline TG (mg/dL) & $139.3 \pm 72.7$ & $149.6 \pm 94.1$ & $137.3 \pm 67.7$ & 0.270 \\
\hline \multicolumn{5}{|l|}{ Post-therapeutic lipid levels } \\
\hline HDL-C (mg/dL) & $49.1 \pm 13.9$ & $50.7 \pm 14.2$ & $48.9 \pm 13.8$ & 0.473 \\
\hline LDL-C (mg/dL) & $109.9 \pm 26.2$ & $120.4 \pm 31.2$ & $108.2 \pm 25.1$ & 0.011 \\
\hline LDL-C/HDL-C & $2.45 \pm 0.89$ & $2.56 \pm 0.88$ & $2.43 \pm 0.90$ & 0.446 \\
\hline $\mathrm{TG}(\mathrm{mg} / \mathrm{dL})$ & $140.3 \pm 62.5$ & $142.5 \pm 62.8$ & $139.9 \pm 62.6$ & 0.821 \\
\hline Follow up period (months) & $51.8 \pm 38.3$ & $32.9 \pm 28.8$ & $55.5 \pm 38.9$ & $<0.001$ \\
\hline Treated lesions & $(\mathrm{n}=418)$ & $(\mathrm{n}=60)$ & $(\mathrm{n}=358)$ & \\
\hline Iliac artery & $228(54.5 \%)$ & $19(31.7 \%)$ & $209(58.4 \%)$ & \\
\hline Femoral artery & $165(39.5 \%)$ & $37(61.7 \%)$ & $128(35.8 \%)$ & $<0.001$ \\
\hline Popliteal artery or below & $25(6.0 \%)$ & $4(6.7 \%)$ & $21(5.9 \%)$ & \\
\hline \multicolumn{5}{|l|}{ The type of lesion } \\
\hline TASC II type A or B & $231(55.3 \%)$ & $25(41.7 \%)$ & $206(57.5 \%)$ & \\
\hline TASC II type C or D & $187(44.7 \%)$ & $35(58.3 \%)$ & $152(42.4 \%)$ & 0.025 \\
\hline \multicolumn{5}{|l|}{ Treatment for vascular lesions } \\
\hline Bypass surgery & $188(45.0 \%)$ & $33(55.0 \%)$ & $155(43.3 \%)$ & \\
\hline Endovascular treatment & $230(55.0 \%)$ & $27(45.0 \%)$ & $203(56.7 \%)$ & 0.095 \\
\hline
\end{tabular}

HTN: hypertension; DM: diabetes mellitus; PCI: percutaneous coronary intervention; CABG: coronary artery bypass grafting; HDL-C: high-density lipoprotein cholesterol; LDL-C: low-density lipoprotein cholesterol; TG: triglyceride

events (restenosis $>75 \%$ in diameter or occlusion) occurred in 60 lesions, including iliac for 19 and infrainguinal lesions for 41 (femoral artery for 37 and popliteal artery or below for 4 lesions), in 51 patients (VE group). Of 60 lesions with local vascular events, 25 lesions (41.7\%) were developed among the TASC II type A or type $\mathrm{B}$ lesions, and 35 lesions (58.3\%) were among the TASC II type C or type D lesions. Local vascular events significantly occurred more frequently in TASC II type $\mathrm{C}$ or D lesions than in type A or B lesions $(P=0.025)$. Regarding the difference in treatments for vascular lesions, 33 lesions (55\%) occurred in patients undergoing surgical procedures and 27 lesions (45\%) in those undergoing EVT ( $P=0.095$; not significant, NS). In univariate analysis of clinical characteristics between the VE and NoVE group, there were no significant differences in age and gender, the prevalence of treatment for HTN or DM, statin drugs prescription, and the prevalence of hemodialysis. Treatment for CAD, including percutaneous coronary intervention (PCI) and coronary artery bypass grafting (CABG), was required for 30 patients (PCI for 27 and $C A B G$ for 3 patients) before or after treatment of PAD. The prevalence of treatment for CAD in the VE group was not significantly different from the prevalence 
in the NoVE group $(P=0.193)$. Patients in the VE group showed a significantly higher post-therapeutic LDL-C level $(120.4 \pm 31.2$ vs. $108.2 \pm 25.1(\mathrm{mg} / \mathrm{dL}) ; P=0.01)$ than those in the NoVE group and a higher prevalence of infrainguinal lesions relative to iliac lesions $(P<0.001)$. Regarding the comparison between the pre- and posttherapeutic serum lipid levels, the post-therapeutic HDLC levels (post-therapeutic $49.1 \pm 13.9$ vs. pre-therapeutic $43.6 \pm 13.0(\mathrm{mg} / \mathrm{dL})$ ), LDL-C levels (post-therapeutic $109.9 \pm 26.2$ vs. pre-therapeutic $116.1 \pm 30.7(\mathrm{mg} / \mathrm{dL}))$ and the LDL-C/HDL-C ratio (post-therapeutic $2.45 \pm 0.89$ vs. pre-therapeutic $2.90 \pm 1.17$ ) were all significantly improved compared to the pre-therapeutic values $(P$ $<0.001)$. The post-therapeutic TG levels $(140.3 \pm 62.5 \mathrm{mg} /$ $\mathrm{dL})$ were not different from the pre-therapeutic levels $(139.3 \pm 72.7 \mathrm{mg} / \mathrm{dL})(P=0.771)$.

The mean follow-up period until the endpoint of this study was $55.5 \pm 38.9$ (range 4 to 120) months for the NoVE group, which was significantly higher than those for the VE group (32.9 \pm 28.8 (range 4 to 113) months) $(P<0.001)$. The difference in the follow-up period between the two groups may result from the presence or absence of local vascular events occurrence, which was the endpoint of this study. There were 32 deaths (10.4\%) during the follow-up period, including deaths from malignancy $(n=5)$, pneumonia $(n=4)$, myocardial infarction and/or heart failure $(n=4)$, sepsis and/or multiple organ failure $(n=2)$, perforation or necrosis of the digestive tract $(\mathrm{n}=2)$, mediastinitis $(\mathrm{n}=1)$, and unknown cause $(n=14)$. These cases were represented as censored cases in the calculation of the Kaplan-Meier method.

As the TASC II type C/D has been reported as a predictor for adverse outcome after treatment of PAD, ${ }^{6,11}$ we examined in more detail the relationship between local vascular events occurrence and the site and type of lesions classified with TASC II guidelines. Table 2 summarizes the number of lesions classified with TASC II according to the type of treatment and the site of the lesion, and the relationship with development of later local vascular events. As shown in Table 2, local vascular events occurred more frequently in TASC II type C/D lesions compared to type A/B lesions in the total patients group (odds ratio $1.90 ; 95 \% \mathrm{CI} 1.09-3.30 ; P=0.025$ ). For comparison of vascular events rate in patients undergoing bypass surgery and EVT individually, difference did not reach statistical significance, probably due to the small number of patients in each group. The prevalence of TASC II type C/D lesions was significantly higher in infrainguinal lesions $(P<0.001)$ and in patients undergo- ing surgery than in those undergoing EVT $(P<0.001)$.

Subsequently, clinical factors that may be related to the development of local vascular events were assessed with multivariate analysis using Cox proportional hazard model with stepwise method to identify correlations with the later events occurrence. The items assessed included male gender (vs. female), surgical treatment (vs. EVT), the presence of $\mathrm{DM}, \mathrm{CAD}$ requiring $\mathrm{PCI}$ or $\mathrm{CABG}$, infrainguinal lesions (vs. iliac lesions), TASC II type C/D lesions (vs. type A/B lesions), pre-therapeutic TG levels $<140$ (mg/dL), and post-therapeutic LDL-C levels $<130$ $(\mathrm{mg} / \mathrm{dL})$, which were selected based on the $\mathrm{p}$ value in the univariate analysis. The cut-off values of LDL-C and TG levels were calculated from receiver-operating characteristics curves (ROC curves) using statistical software. The results are shown in Table 3, which indicated that the infrainguinal lesions relative to iliac lesions significantly increased in the risk for later, local vascular events (hazard ratio 3.38; 95\%CI 1.60-7.10; $P=0.001$ ) and the lowered LDL-C levels $<130(\mathrm{mg} / \mathrm{dL})$ decreased in the risk for later vascular events (hazard ratio $0.34 ; 95 \% \mathrm{CI}$ $0.17-0.67 ; P=0.002)$. In contrast, gender male $(P=0.550)$, surgical treatment relative to EVT $(P=0.731)$, presence of DM $(P=0.865)$, CAD requiring PCI or CABG $(P=0.129)$, TASC II type $\mathrm{C} / \mathrm{D}$ (vs. type A/B) $(P=0.937)$, and pre-therapeutic TG levels $<140(\mathrm{mg} / \mathrm{dL})(P=0.966)$ did not significantly influence the risk for later vascular events.

Based on the results from multivariate analysis, we compared the rate of freedom from later local vascular events (VE-free rate) between the two groups divided according to the post-therapeutic LDL-C level. Figure 1 shows a comparison of Kaplan-Meier curves indicating the VE-free rate, with a significant difference between the two patient groups. Patients with post-therapeutic LDL-C levels $<130 \mathrm{mg} / \mathrm{dL}$ had a significantly better VEfree rate $(P=0.005$; by the logrank test). A cumulative rate free from vascular events was approximately more than $90 \%$ for the 5 year and more than $80 \%$ for the 10 year in those with post-therapeutic LDL-C levels $<130 \mathrm{mg} / \mathrm{dL}$. The rate was, however, lower in those with post-therapeutic LDL-C levels $>130 \mathrm{mg} / \mathrm{dL}$, which was less than $80 \%$ for the 5 year and 10 year. Onset of vascular events, which was defined as the endpoint of the study in the VE group, was $32.9 \pm 28.8$ (months) as described previously and shown in Table 1. 
Table 2 The number of lesions classified with TASC II according to the type of treatment and site of lesion, and the relationship with development of later vascular events

\begin{tabular}{|c|c|c|c|c|c|}
\hline & & \multicolumn{2}{|c|}{ The number of lesion classified with TASC II } & \multirow{2}{*}{$P$ value } & \multirow{2}{*}{$\begin{array}{l}\text { Odds ratio } \\
(95 \% \mathrm{CI})\end{array}$} \\
\hline & & Type A or B & Type C or D & & \\
\hline Iliac lesion & & 168 & 60 & & \\
\hline Infrainguinal lesion & & 63 & 127 & $<0.001$ & $5.64(3.70-8.61)$ \\
\hline \multicolumn{6}{|c|}{ Local vascular events (total) } \\
\hline & Yes & 25 & 35 & & \\
\hline & No & 206 & 152 & 0.025 & $1.90(1.09-3.30)$ \\
\hline \multicolumn{6}{|c|}{ Local vascular events in patients undergoing bypass surgery } \\
\hline \multirow[t]{2}{*}{ Iliac lesion } & Yes & 0 & 4 & & \\
\hline & No & 6 & 55 & & \\
\hline \multirow[t]{2}{*}{ Infrainguinal lesion } & Yes & 0 & 29 & & \\
\hline & No & 5 & 89 & & \\
\hline \multirow[t]{2}{*}{ total } & Yes & 0 & 33 & & \\
\hline & No & 11 & 144 & 0.217 & $\begin{array}{l}\text { N/A (due to data } \\
\text { containing zero) }\end{array}$ \\
\hline \multicolumn{6}{|c|}{ Local vascular events in patients undergoing EVT } \\
\hline \multirow[t]{2}{*}{ Iliac lesion } & Yes & 15 & 0 & & \\
\hline & No & 147 & 1 & & \\
\hline \multirow[t]{2}{*}{ Infrainguinal lesion } & Yes & 10 & 2 & & \\
\hline & No & 48 & 7 & & \\
\hline \multirow[t]{2}{*}{ total } & Yes & 25 & 2 & & \\
\hline & No & 195 & 8 & 0.332 & $1.95(0.39-9.70)$ \\
\hline \multicolumn{6}{|c|}{ Comparison of the number of patients between bypass surgery and EVT according to the TASC II classification } \\
\hline bypass surgery & & 11 & 177 & & \\
\hline EVT & & 220 & 10 & $<0.001$ & $354.0(147-852)$ \\
\hline
\end{tabular}

EVT: endovascular treatment; N/A: not available

Table 3 Relationship between risk factors and later vascular events by Cox proportional hazard analysis

\begin{tabular}{lccc}
\hline & Hazard Ratio & $95 \%$ CI & \\
\hline Clinical factors & & & 0.554 \\
Gender, male (vs. female) & 0.77 & $0.33-1.81$ & 0.317 \\
Surgical treatment (vs. EVT) & 1.42 & $0.72-2.80$ & 0.867 \\
Diabetes mellitus & 1.06 & $0.55-2.04$ & 0.128 \\
CAD requiring PCI or CABG & 0.21 & $0.03-1.56$ & 0.001 \\
Infrainguinal (vs. iliac) lesion & 3.35 & $1.63-6.90$ & 0.937 \\
TASC II type C/D (vs. type A/B) & 0.93 & $0.17-5.01$ & 0.969 \\
Pre-therapeutic TG $<140$ & 0.99 & $0.51-1.92$ & 0.002 \\
Post-therapeutic LDL-C $<130$ & 0.34 & $0.17-0.67$ & 0.002 \\
\hline
\end{tabular}

CI: confidence interval; EVT: endovascular treatment; PCI: percutaneous coronary intervention; CABG: coronary artery bypass grafting; LDL-C: low-density lipoprotein cholesterol; TG: triglyceride 


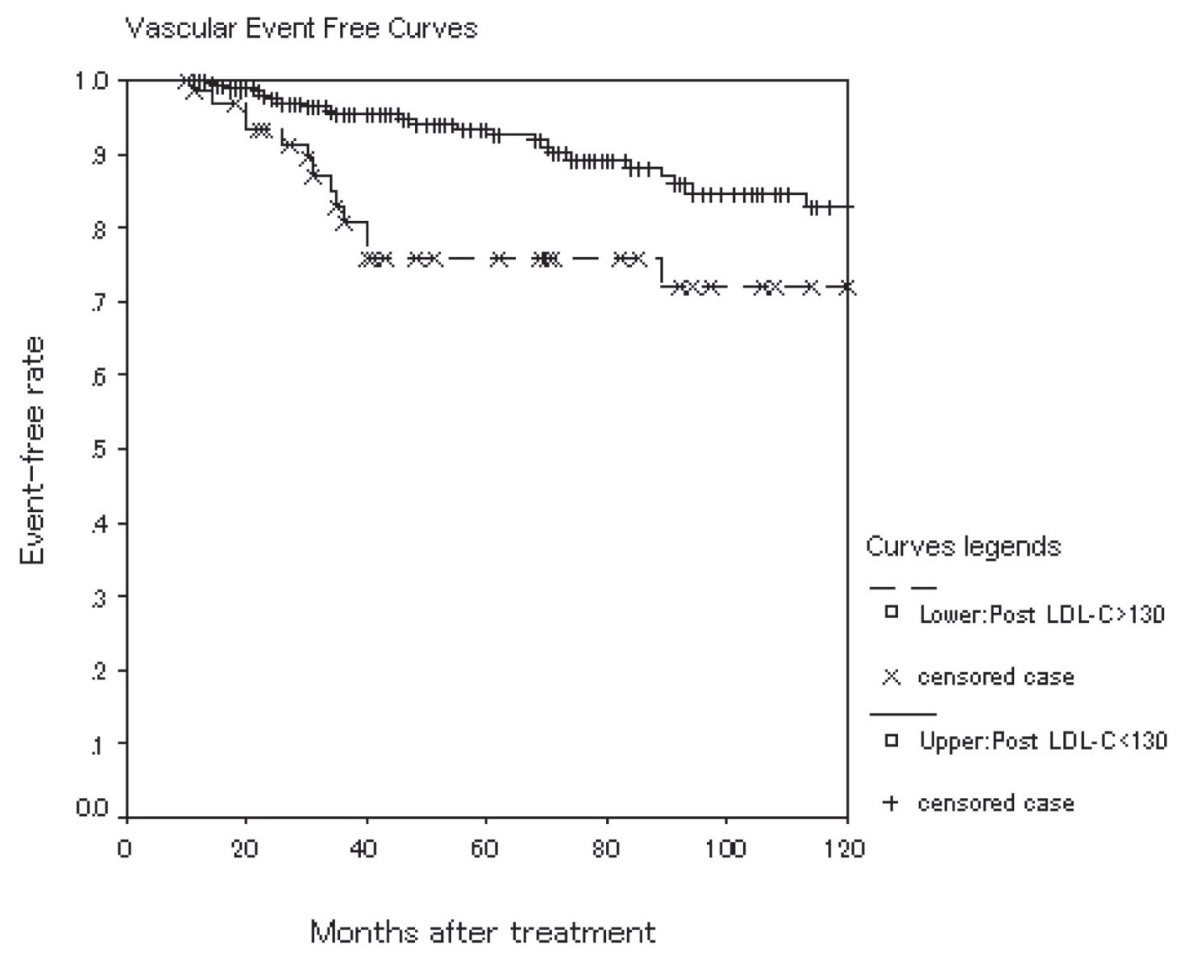

Fig. 1 Kaplan-Meier curves indicating freedom from local vascular events in the group of patients with post-therapeutic LDL-C $<130$ and those with LDL-C $>130$ (mg/dL)

\section{Discussion}

Our data indicated that later, local vascular events after surgical or endovascular treatment for PAD may occur more frequently in patients treated for infrainguinal lesions and those with post-therapeutic mean LDL-C levels $>130(\mathrm{mg} / \mathrm{dL})$, at an approximate hazard ratio of 3.0 to 3.5. PAD is a part of clinical manifestation of systemic atherosclerosis, and the presence of PAD elevates the risk for cardiovascular events due to atherosclerosis, including myocardial infarction and stroke. ${ }^{2,3,5)}$ Thus, of course, the prognosis of patients with PAD depends on the prevention of these systemic cardiovascular events, rather than on the presence or absence of local vascular events. However, local vascular events, such as restenosis and occlusion, may elevate the risk for development of CLI and amputation, and the reported annual mortality rate is as high as $25 \%$ in patients with CLI and in those undergoing major amputation. ${ }^{6,14)}$ Therefore, therapeutic strategy for preventing local vascular events may also contribute to the improvement of life prognosis in patients with PAD.

There have been a number of reports describing the value of using statin drugs in patients with PAD, including all cause and cardiac mortality. ${ }^{3,15,16)}$ LDL lowering therapy has been reported to improve symptoms of intermittent claudication and pain-free walking performance, and has also been reported to reduce the risk of new or worsening claudication in patients with PAD. ${ }^{6,17)}$ In the Heart Protection Study of cholesterol lowering with simvastatin, there was a $16 \%$ relative reduction in the rate of a first peripheral vascular event, primarily due to a $20 \%$ relative reduction in noncoronary revascularization procedures. ${ }^{13)}$ This study suggests that it may be the pleiotropic effects of statins that are responsible for benefit in PAD, including not only lipid-lowering effects but also anti-inflammatory effects. Statins have been also reported to reduce the expression of matrix metalloproteinases independently of their cholesterol-lowering effect. ${ }^{6}$ However, the potential benefits of LDL lowering with statins on the underlying physiology in PAD remain incompletely understood.

The lipid level per se on individual cases may vary with pre-therapeutic lipid levels, racial variation, and individual lifestyle and comorbidity. There have been few reports describing the direct relationship between the 
serum lipid levels and later vascular events, especially in patients undergoing surgical treatment. ${ }^{6}$ ) This is probably due to that those with PAD generally have a low chance of long-term survival, ${ }^{5,6}$ ) which would inhibit the practice of this type of study. Furthermore, therapeutic strategy for patients with PAD has been changed in response to progress in EVT and publication of TASC and TASC II guidelines. ${ }^{910)}$ The majority of TASC II type A and type $\mathrm{B}$ lesions in iliac arteries are currently treated with EVT, and the number of patients undergoing open surgical procedures has been diminished. Thus, results from the present study including surgical cases are thought to be of significance. The cut-off value of LDL-C levels $<130 \mathrm{mg} / \mathrm{dL}$ in this study was not a strict target value, as recent literature including AHA/ACC guidelines described that LDL-C should be $<100 \mathrm{mg} / \mathrm{dL}$ for all patients with $\mathrm{CAD}$ and other clinical forms of atherosclerotic disease, and even $<70 \mathrm{mg} / \mathrm{dL}$ in patients at the highest risk. ${ }^{18,19)}$ The optimal target LDL-C levels in Japanese patients with atherosclerotic disease may be different, ${ }^{20,21)}$ but later local vascular events occurrence showed a significant difference between patients with LDL-C levels $<130$ and those with LDL-C levels $>130 \mathrm{mg} / \mathrm{dL}$ in this study. The target value of LDL$\mathrm{C}$ levels $<130 \mathrm{mg} / \mathrm{dL}$ seems to be the minimum requirement in the treatment of Japanese patients with PAD to reduce local vascular events.

Regarding the comparison between the pre- and posttherapeutic serum lipid levels, the post-therapeutic HDL$\mathrm{C}, \mathrm{LDL}-\mathrm{C}$, and LDL-C/HDL-C ratio were all significantly improved compared to pre-therapeutic values. This was probably due to the management of atherosclerotic risk factors including statin treatment, although aggressive lipid-modifying medications were not always performed during the follow-up period. Lifestyle guidance like diet and exercise therapy has been intensively conducted by medical personnel in the outpatient clinic throughout the follow-up period, which may have contributed to the improvement of lipid levels.

Our study also indicates that patients with infrainguinal lesions are at high risk for later local vascular events occurrence. As shown in Table 2, TASC II type C/D lesions comprised 127 of 180 (70.6\%) infrainguinal lesions, whereas, 60 of 228 (26.3\%) iliac lesions. The prevalence of the type C/D lesion, which has been reported to significantly influence graft patency in surgical cases, was further higher in infrainguinal lesions $(P<0.001)$. The difference in the prevalence of the type $\mathrm{C} / \mathrm{D}$ lesion may account for the high risk for later local vascular events occurrence in patients with infrainguinal lesions, but the Cox proportional hazard analysis did not indicate the TASC type C/D as a significant predictor for total later vascular events in our study. The disparity between the results from univariate analysis and the Cox proportional hazard analysis may be due to the onset time of vascular events, as the latter analysis investigated the effects of predictor over the time course.

The subjects in this study were limited to those under the age of 70 to ensure sufficient follow-up period and to minimize the influence of aging on local vascular events. In younger patients presenting with IC, however, the value of surgical revascularization remains to be elucidated. ${ }^{22,23)}$ Reed and colleagues reported that the 5 year primary patency rate after aortobifemoral bypass was significantly lower in patients aged 50 or younger than in those over the age of 50. ${ }^{23)}$ In the report by Green and colleagues, patency rates of primary prosthetic aboveknee femoropopliteal bypass grafts at 5 years were lower in patients aged 65 or younger. ${ }^{22)}$ The underlying pathogenesis in these younger patients would be the presence of multiple atherosclerotic risk factors including dyslipidemia, which may account for the development of PAD in younger patients. Risk reductions in atherosclerotic factors among younger patients would be an important issue for the improvement of prognosis of overall patients with PAD.

The present study did not examine later cardiovascular events such as myocardial infarction and stroke, but limited to local vascular events after treatment of PAD. Lipidmodifying therapy in those with PAD, however, would be highly beneficial in the prevention of later cerebrovascular or coronary events. For the primary prevention of atherosclerotic disease like CAD, the importance of adequate control of dyslipidemia has been reported, and lipid-modifying therapies like statin treatment have an established role in the treatment of occlusive atherosclerotic disease. ${ }^{18,24)}$ In general, LDL-C at a high serum level may accumulate in the artery wall, where they are oxidized and taken up by foam cells, in a process that leads to the development and progression of atherosclerosis. ${ }^{24)} \mathrm{HDL}-$ $\mathrm{C}$ is reported to oppose atherosclerosis by removing cholesterol from foam cells, by inhibiting the oxidation of LDL-C, and by limiting the inflammatory processes that underlie atherosclerosis. ${ }^{24)}$ As previously described, the definite prognosis of patients with PAD depends on the prevention of fatal CAD and cerebrovascular disease. In patients with AAA, our group has reported the relationship between the lipid levels and later cardiovascular 
events after surgical treatment for AAA. ${ }^{25)}$ The relationship between lipid levels and later cardiovascular events in patients with PAD would be also worth reporting.

There are some limitations in this study. In our study design, patients with PAD receiving surgical or endovascular treatment were retrospectively divided into two groups, based on the patient outcome of whether they would have local vascular events later or not, as a single center experience. Lipid-modifying therapy was not given to all patients. Second, the findings of our study were limited to those aged $<70$ at surgical or endovascular treatment; thus the value of lipid-modifying therapy in patients with age older than the present study group is unknown. Since the majority of subjects were male patients, we can draw no conclusions on the association of lipids with local vascular events in women. Third, the follow-up period was relatively short in some patients; thus, the short follow-up period may influence the incidence of local vascular events. Although there are many limitations, our study results are not so inconsistent with previous reports and may help us to prognosticate the outcome at least in part of patients with PAD.

In conclusion, our study indicated that an increase in the post-therapeutic LDL-C levels $>130 \mathrm{mg} / \mathrm{dL}$ and the presence of infrainguinal lesions were significant predictors for the development of later local vascular events during follow-up after surgical or endovascular treatment for PAD. Patients at high risk for later, local vascular events should require a careful follow-up to prevent the development of CLI or amputation, and may also require an aggressive lipid-modifying therapy.

\section{REFERENCES}

1) Diehm C, Schuster A, Allenberg JR, et al. High prevalence of peripheral arterial disease and comorbidity in 6880 primary care patients: cross-sectional study. Atherosclerosis 2004; 172: 95-105. [Medline] [CrossRef]

2) Murabito JM, Evans JC, Nieto K, et al. Prevalence and clinical correlates of peripheral arterial disease in the Framingham Offspring Study. Am Heart J 2002; 143: 961-5. [Medline] [CrossRef]

3) Schanzer A, Hevelone N, Owens C, et al. Statins are independently associated with reduced mortality in patients undergoing infrainguinal bypass graft surgery for critical limb ischemia. J Vasc Surg 2008; 47: 77481. [Medline] [CrossRef]

4) Selvin E, Erlinger TP. Prevalence of and risk factors for peripheral arterial disease in the United States. Circulation 2004; 110: 738-43. [Medline] [CrossRef]

5) Criqui MH, Langer RD, Fronek A, et al. Mortality over a period of 10 years in patients with peripheral arterial disease. N Engl J Med 1992; 326: 381-6. [Medline] [CrossRef]

6) Hirsch AT, Haskal ZJ, Hertzer NR, et al. ACC/AHA 2005 guidelines for the management of patients with peripheral arterial disease (lower extremity, renal, mesenteric, and abdominal aortic): executive summary a collaborative report from the American Association for Vascular Surgery/Society for Vascular Surgery, Society for Cardiovascular Angiography and Interventions, Society for Vascular Medicine and Biology, Society of Interventional Radiology, and the ACC/ AHA Task Force on Practice Guidelines (Writing Committee to Develop Guidelines for the Management of Patients With Peripheral Arterial Disease) endorsed by the American Association of Cardiovascular and Pulmonary Rehabilitation; National Heart, Lung, and Blood Institute; Society for Vascular Nursing; TransAtlantic Inter-Society Consensus; and Vascular Disease Foundation. Circulation 2006; 113: e463-654. [Medline]

7) 2011 ACCF/AHA Focused Update of the Guideline for the Management of Patients With Peripheral Artery Disease (Updating the 2005 Guideline). Circulation 2011; 124: 2020-45. [Medline] [CrossRef]

8) Henke PK, Blackburn S, Proctor MC, et al. Patients undergoing infrainguinal bypass to treat atherosclerotic vascular disease are underprescribed cardioprotective medications: effect on graft patency, limb salvage, and mortality. J Vasc Surg 2004; 39: 357-65. [Medline] [CrossRef]

9) Dormandy JA, Rutherford RB. Management of peripheral arterial disease (PAD). TASC Working Group. TransAtlantic Inter-Society Consensus (TASC). J Vasc Surg 2000; 31(1 Pt 2): S1-296. [Medline]

10) Norgren L, Hiatt WR, Dormandy JA, et al. Inter-Society Consensus for the Management of Peripheral Arterial Disease (TASC II). J Vasc Surg 2007; 45 Suppl S: S5-67.

11) Kudo T, Chandra FA, Ahn SS. Long-term outcomes and predictors of iliac angioplasty with selective stenting. J Vasc Surg 2005; 42: 466-75. [Medline] [CrossRef]

12) Kumada $Y$, Aoyama $T$, Ishii $H$, et al. Long-term outcome of percutaneous transluminal angioplasty in chronic haemodialysis patients with peripheral arterial disease. Nephrol Dial Transplant 2008; 23: 3996-4001. [Medline] [CrossRef]

13) Heart Protection Study Collaborative Group MRC/ BHF Heart Protection Study of cholesterol lowering with simvastatin in 20,536 high-risk individuals: a randomised placebo-controlled trial. Lancet 2002; 360(9326): 7-22. [Medline] [CrossRef]

14) Feinglass J, Pearce WH, Martin GJ, et al. Postoperative and late survival outcomes after major amputation: findings from the Department of Veterans Affairs National Surgical Quality Improvement Program. Surgery 2001; 130: 21-9. [Medline] [CrossRef] 
15) Coppola G, Novo S. Statins and peripheral arterial disease: effects on claudication, disease progression, and prevention of cardiovascular events. Arch Med Res 2007; 38: 479-88. [Medline] [CrossRef]

16) Erez G, Leitersdorf E. The rationale for using HMGCoA reductase inhibitors ('statins') in peripheral arterial disease. Eur J Vasc Endovasc Surg 2007; 33: 192-201. [Medline] [CrossRef]

17) Mohler ER III, Hiatt WR, Creager MA, Study Investigators Cholesterol reduction with atorvastatin improves walking distance in patients with peripheral arterial disease. Circulation 2003; 108: 1481-6. [Medline] [CrossRef]

18) LaRosa JC, Grundy SM, Waters DD, et al. Intensive Lipid lowering with atorvastatin in patients with stable coronary disease. N Engl J Med 2005; 352: 1425-35. [Medline] [CrossRef]

19) Smith SC, Benjamin EJ, Bonow RO, et al. AHA/ACCF secondary prevention and risk reduction therapy for patients with coronary and other atherosclerotic vascular disease: 2011 Update: a guideline from the American Heart Association and American College of Cardiology Foundation. Circulation 2011; 124: 245873. [Medline] [CrossRef]

20) Mabuchi H, Kita T, Matsuzaki M, et al. Large scale cohort study of the relationship between serum cholesterol concentration and coronary events with low- dose simvastatin therapy in Japanese patients with hypercholesterolemia and coronary heart disease: secondary prevention cohort study of the Japan Lipid Intervention Trial (J-LIT). Circ J 2002; 66: 1096-100. [Medline] [CrossRef]

21) Matsuzaki M, Kita T, Mabuchi H, et al. Large scale cohort study of the relationship between serum cholesterol concentration and coronary events with lowdose simvastatin therapy in Japanese patients with hypercholesterolemia. Circ J 2002; 66: 1087-95. [Medline] [CrossRef]

22) Green RM, Abbott WM, Matsumoto T, et al. Prosthetic above-knee femoropopliteal bypass grafting: five-year results of a randomized trial. J Vasc Surg 2000; 31: 417-25. [Medline] [CrossRef]

23) Reed AB, Conte MS, Donaldson MC, et al. The impact of patient age and aortic size on the results of aortobifemoral bypass grafting. J Vasc Surg 2003; 37: 1219-25. [Medline] [CrossRef]

24) Barter P. The role of HDL-cholesterol in preventing atherosclerotic disease. Eur Heart J 2005; 7(suppl F): F4-8. [CrossRef]

25) Takahashi J, Wakamatsu Y, Ishii K, et al. Preoperative HDL-C predicts later cardiovascular events after abdominal aortic aneurysm surgery. Ann Vasc Dis 2011; 4: 115-20. [CrossRef] 